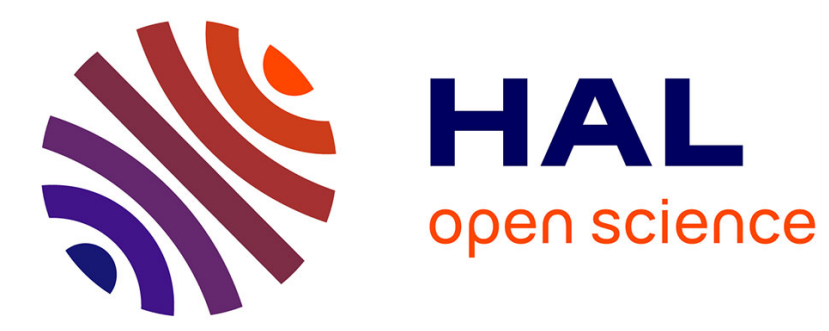

\title{
On the Horton-Rogers-Lapwood convective instability with vertical vibration: Onset of convection
}

Gérald Bardan, Abdelkader Mojtabi

\section{To cite this version:}

Gérald Bardan, Abdelkader Mojtabi. On the Horton-Rogers-Lapwood convective instability with vertical vibration: Onset of convection. Physics of Fluids, 2000, 12 (11), pp.2723-2731. 10.1063/1.1313551 . hal-01871723

\section{HAL Id: hal-01871723 \\ https://hal.science/hal-01871723}

Submitted on 11 Sep 2018

HAL is a multi-disciplinary open access archive for the deposit and dissemination of scientific research documents, whether they are published or not. The documents may come from teaching and research institutions in France or abroad, or from public or private research centers.
L'archive ouverte pluridisciplinaire HAL, est destinée au dépôt et à la diffusion de documents scientifiques de niveau recherche, publiés ou non, émanant des établissements d'enseignement et de recherche français ou étrangers, des laboratoires publics ou privés. 


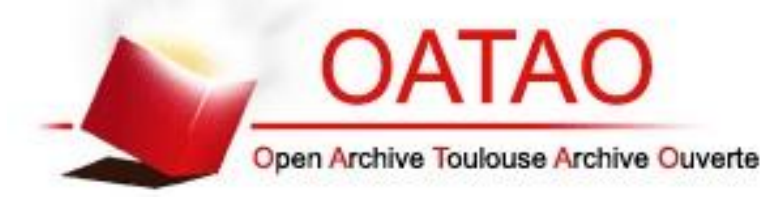

Open Archive Toulouse Archive Ouverte

OATAO is an open access repository that collects the work of Toulouse researchers and makes it freely available over the web where possible

This is an author's version published in: http://oatao.univ-toulouse.fr/20633

\section{Official URL:}

http://doi.org/10.1063/1.1313551

\section{To cite this version:}

Bardan, Gérald and Mojtabi, Abdelkader On the Horton-

Rogers-Lapwood convective instability with vertical vibration: Onset of convection. (2000) Physics of Fluids, 12 (11). 27232731. ISSN 1070-6631

Any correspondence concerning this service should be sent to the repository administrator: tech-oatao@listes-diff.inp-toulouse.fr 


\title{
On the Horton-Rogers-Lapwood convective instability with vertical vibration: Onset of convection
}

\author{
G. Bardan and A. Mojtabi \\ U.F.R. M.I.G. bat. 1 R2 U.M.R. 5502, Département de Mécanique, Université Paul Sabatier, 118, \\ Route de Narbonne, 31062 Toulouse Cedex, France
}

(Received 5 January 2000; accepted 26 July 2000)

\begin{abstract}
We present a numerical and analytical study of diffusive convection in a rectangular saturated porous cell heated from below and subjected to high frequency vibration. The configuration of the Horton-Rogers-Lapwood problem is adopted. The classical Darcy model is shown to be insufficient to describe the vibrational flow correctly. The relevant system is described by time-averaged Darcy-Boussinesq equations. These equations possess a pure diffusive steady equilibrium solution provided the vibrations are vertical. This solution is linearly stable up to a critical value of the stability parameter depending on the strength of the vibration. The solutions in the neighborhood of the bifurcation point are described analytically as a function of the strength of vibration, and the larger amplitude states are computed numerically using a spectral collocation method. Increasing the vibration amplitude delays the onset of convection and may even create subcritical solutions. The majority of primary bifurcations are of a special type of symmetry-breaking bifurcation even if the system is subjected to vertical vibration. (C) 2000 American Institute of Physics. [S1070-6631(00)00411-6]
\end{abstract}

\section{INTRODUCTION}

Natural convection flow in porous media due to thermal buoyancy has been widely studied by Bories ${ }^{1}$ and well documented in the literature in the excellent book recently written by Nield and Bejan, ${ }^{2}$ while only a few works have been devoted to thermovibrational convection in porous media $\left(\right.$ Gershuni and Lyubimov ${ }^{3}$ ). Vibration of a cell containing a saturated porous medium in the presence of a temperature gradient can give rise to vibrational convection. This convection mechanism also operates under conditions of weightlessness, when there is no static gravity field.

The present study is concerned with the bifurcation structure associated with steady thermovibrational convection in a finite two-dimensional saturated porous cavity heated from below.

Beck $^{4}$ seems to have been the first to consider convection in a finite three-dimensional horizontal box of porous material saturated with fluid and heated from below. Using linear stability analysis, he found the critical Rayleigh number for the onset of convection in a box. In the present twodimensional study we effectively restrict ourselves to thin three-dimensional boxes with length greater than or equal to the height. For three-dimensional boxes, numerical solutions based on the Galerkin method have been reported by Straus and Schubert, ${ }^{5-7}$ Schubert and Straus, ${ }^{8}$ and Caltagirone et al. ${ }^{9}$ The number of numerical investigations into twodimensional large amplitude convection is also substantial and we refer to the work of Riley and Winters. ${ }^{10}$ Their main results concern a nonlinear study and mode interactions. They also deal with linear stability. For a cavity of fixed aspect ratio, an analytical linear stability study shows that a countably infinite set of eigenmodes exists. These eigenmodes occur at discrete eigenvalues and represent convective flows with $i$ and $j$ rolls in the horizontal and vertical directions, respectively. The preferred mode of convection (the one with the lowest critical Rayleigh number) always has one cell in the vertical direction: The number of cells in the horizontal direction depends upon the aspect ratio. They also emphasize that there is a close analogy between the porous medium case and the pure fluid case, which is generally known as the Rayleigh-Bénard problem. They share $Z_{2}$ $\times Z_{2}$ symmetry, but the porous medium problem possesses in addition a translational invariance so that a two-cell solution in a cavity of aspect ratio 2 is equivalent to a four-cell solution in a cavity of aspect ratio 4, and so on. This translational invariance arises from the slip boundary conditions which apply at the boundaries of the porous medium.

In this paper we present an extensive discussion of the effect of high-frequency vibration on the previous problem.

High frequency periodic accelerations occurring on microgravity platforms ( $g$-jitter), may induce disturbances in space experiments that deal with liquids in the presence of density gradients. In the low-gravity environment of space stations, buoyancy-induced convection may be expected to be minimized; however, $g$-jitter induced by onboard machinery or excited by crew activities can result in convection in fluids when there are density gradients. These convective effects may have an influence on fluid or porous medium science experiments or on material processing as, for example, solidification processes. The $g$-jitter is modeled by a unidirectional, harmonically oscillating, small-amplitude acceleration field (Alexander ${ }^{11}$ ). Since the governing equations are nonlinear, the fluctuating acceleration with zero average induces a periodic response consisting of a steady solution with a nonzero mean superimposed on a time-dependent, zero-average fluctuation (Gershuni and Zhukhovitsky ${ }^{12}$ ). The averaged field, also referred to in the literature as streaming 


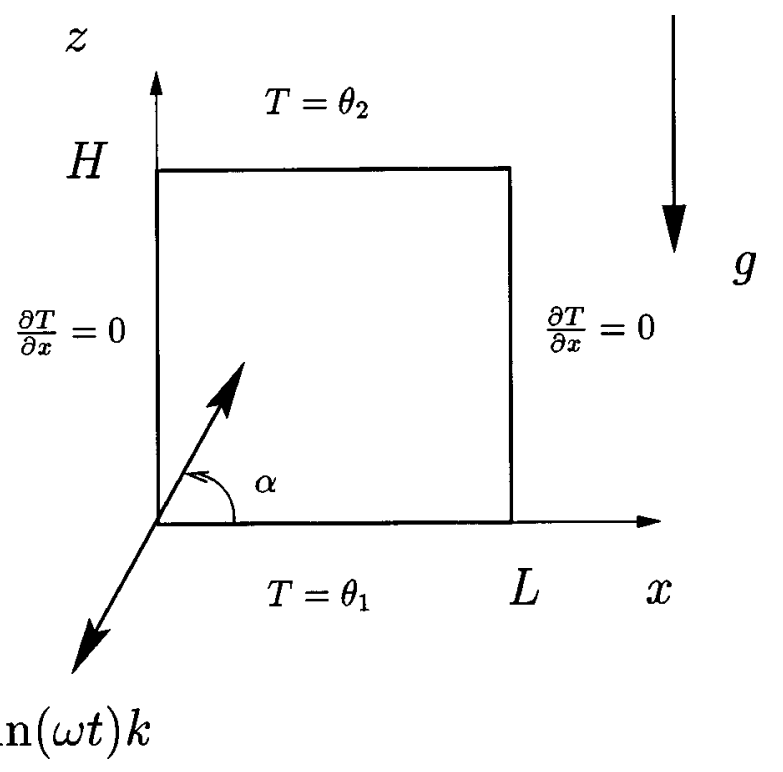

FIG. 1. Geometrical configuration and axis of coordinates. Sketch of the cavity configuration.

flow, is significant because it can give a relevant contribution to the heat transfer in a time-averaged sense.

Studies on thermal convection in a gravitationally modulated fluid layer with rigid, isothermal boundaries heated either from below or from above (i.e., for the RayleighBénard problem) include the early work by Gresho and Sani ${ }^{13}$ and Rosenblat and Tanaka. ${ }^{14}$ More recently, this work has been extended by Roppo et al.,${ }^{15}$ Halers et al. ${ }^{16}$ and Hohenberg and Swift. ${ }^{17}$ A number of experiments, in reasonable agreement with the theoretical predictions, have been performed by Halers et al. ${ }^{18}$ and Niemela and Donnelly. ${ }^{19}$ They find that vertical vibrations stabilize the diffusive solution, i.e., the value of the critical Rayleigh number increases with the strength of vibration.

To our knowledge, only a few works on the effect of vibration have been performed for an enclosure filled by pure fluid. Bardan et al. ${ }^{20}$ present the effect of high frequency jitter in a differentially heated cavity situation and Farooq and Homsy ${ }^{21}$ and Grassia and Homsy ${ }^{22}$ study the effects of low frequency jitter on streaming and buoyant flows.

The effect of vibration on a porous medium is also of interest for the petroleum research community. Vibration production technology is a simple, economic, and efficient way to increase oil production in some oil fields. ${ }^{23}$ Khallouf et al. $^{24}$ made a numerical study of some properties of convective oscillations in porous medium. This study was restricted to the case of finite frequencies. In the limiting case of high frequencies, they found that the effect of vibration disappeared as a result of the simplification adopted in the Darcy model. In this paper, we present a first discussion on the effect of vibration on a porous medium confined in an enclosure heated from below. We will see that a classical Darcy model is not suitable for taking into account the effect of vibration. A mean flow formulation for an adapted Darcy model is proposed. The linear stability problem is solved by means of a Galerkin method. The amplitude equation at the onset of the preferred mode is given.

\section{MATHEMATICAL MODEL AND BASIC EQUATIONS}

We consider a fluid-saturated porous medium enclosed in a container of height $H$ and length $L$. Figure 1 represents the flow configuration and coordinate system. The flow domain is $(x, z) \in \Omega=[0, L] \times[0, H]$. The horizontal walls at $z$ $=0$ and $z=H$ are kept at constant and uniform temperatures $\theta_{1}$ and $\theta_{2}$, respectively. The situation where $\theta_{1}>\theta_{2}$ is adopted. The vertical walls (at $x=0$ and $x=L$ ) are insulated. All the boundaries are assumed rigid. The cavity with its boundaries is subjected to linear harmonic oscillations.

\section{A. Approximations}

(1) The solid matrix is homogeneous, nondeformable, and chemically inert with respect to the fluid.

(2) The fluid is single phase and Newtonian.

(3) The Darcy number is small so that the inertia terms may be neglected.

(4) The solid and fluid phases are in thermal equilibrium.

(5) The relative temperature differences are small enough for the Boussinesq approximation to hold.

(6) Thermal dissipation is negligible and an effective thermal conductivity may be assumed.

(7) The vibration frequency is large and the displacement amplitude is small enough for the averaging method to hold.

\section{B. The mathematical model}

The saturated porous medium in the cavity is considered to satisfy the Boussinesq approximation. The thermophysical properties are constant except for the density in the buoyancy term, which depends linearly on the local temperature. The equation of state has the following form:

$$
\rho(\theta)=\rho_{\text {ref }}\left(1-\beta_{\theta}\left(\theta-\theta_{\text {ref }}\right)\right),
$$

where $\rho_{\text {ref }}=\rho\left(\theta_{\text {ref }}\right)$ and $\beta_{T}=-1 / \rho_{\text {ref }} \partial \rho / \partial \theta$ are the density at standard temperature $\theta_{\text {ref }}=\theta_{2}$ and the thermal expansion coefficient, respectively. By introducing the appropriate coordinates connected with oscillating systems, the gravitational field is replaced by the sum of the gravitational and the vibrational acceleration (2) in the momentum equation

$$
\mathbf{g} \rightarrow-g \mathbf{z}+b \omega^{2} \sin (\omega t) \mathbf{k},
$$

where $\mathbf{k}=\cos \alpha \mathbf{x}+\sin \alpha \mathbf{z}$ is the unit vector along the axis of vibration and $\alpha=(\mathbf{x}, \mathbf{k})$ is the angle of vibration, $b$ is the displacement amplitude, and $\omega$ the angular frequency.

These hypotheses lead to the following dimensionless conservation equations for mass (3), momentum (4), and energy (5), with the Boussinesq approximation. Using the velocity $\mathbf{u}$, the pressure $p$, and the temperature $\theta$ as independent variables, the nondimensional equations of the DarcyBoussinesq model are

$$
\begin{aligned}
& \boldsymbol{\nabla} \cdot \mathbf{u}=0, \\
& \mathbf{u}+A \frac{\partial \mathbf{u}}{\partial t}=-\nabla p+\operatorname{Ra} \theta \mathbf{z}-\operatorname{Ra} R \theta \sin (\hat{\omega} t) \mathbf{k},
\end{aligned}
$$




$$
\frac{\partial \theta}{\partial t}+(\mathbf{u} \cdot \boldsymbol{\nabla}) \theta=\nabla^{2} \theta .
$$

In Eqs. (3)-(5), using the thermal diffusion time as the time-scale factor, lengths are nondimensionalized by $H$, velocity by $a / H$ ( $a$ is the thermal diffusivity), time by $H^{2} / a, \omega$ by $a / H^{2}$, and temperature relative to $\theta_{2}$ by $\theta_{1}-\theta_{2}$. In line with the problem description, the corresponding nondimensional forms of the boundary conditions are

$\mathbf{u} \cdot \mathbf{n}=0 \quad$ on $\partial \Omega \quad(\mathbf{n}$ is the outside normal vector $)$,

$$
\begin{aligned}
& \theta=1 \text { for } z=0, \forall x \\
& \theta=0 \text { for } z=1, \forall x \\
& \frac{\partial \theta}{\partial x}=0 \text { for } x=0 \text { and } A_{L}, \forall z .
\end{aligned}
$$

The problem includes five nondimensional numbers; the Rayleigh number $\mathrm{Ra}=g \beta_{\theta} K\left(\theta_{1}-\theta_{2}\right) H / \nu a$ ( $K$ is the permeability), the $g$-number $R=b \omega^{2} / g$, the pulsation $\hat{\omega}$ $=\omega H^{2} / a, A=\mathrm{Da} M / \epsilon \operatorname{Pr}$ and the aspect ratio $A_{L}=L / H$. Pr $=\nu / a$ is the Prandtl number defined as the ratio between the thermal and viscous diffusive times ( $\nu$ and $a$ are the kinematic and thermal diffusivity, respectively). $\mathrm{Da}=K / H^{2}$ is the Darcy number, $M$ the volumetric specific heat ratio, and $\epsilon$ the porosity. As $A$ is of the order $10^{-6}$ in classical porous media, the contribution of the term $A \partial \mathbf{u} / \partial t$ in Eq. (4) can be neglected but with high-frequency vibration this simplification cannot be made. The term $\sin (\omega t)$ allows very large variation of $\partial \mathbf{u} / \partial t$ and $A \partial \mathbf{u} / \partial t=O(\mathbf{u})$.

\section{The averaged flow equations}

In the asymptotic case of high-frequency oscillations where the period of the displacement $\tau=2 \pi / \omega$ is very low compared to the characteristic times of thermal and kinematic diffusion $\left(\tau \ll H^{2} / a\right.$ and $\tau \ll H^{2} / \nu$ ) the application of the averaging method of Bogljubov and Mittropolskil ${ }^{25}$ only allows the mean flow and heat transfer to be solved. The basic idea consists in treating the periodic flow with an approach similar to that used in studying turbulent flows. The quantities under investigation are divided into two parts: the first, denoted $F=\bar{f}$ (time independent for a $\tau$-periodic behavior), varies slowly with time and the second, denoted $f^{\prime}(\tau$ periodic), varies rapidly with time. Thus we have the following decomposition:

$$
\begin{aligned}
& \mathbf{u}=\mathbf{U}(t)+\mathbf{u}^{\prime}(\omega t), \\
& \theta-\theta_{2}=T(t)+\theta^{\prime}(\omega t), \\
& p=P(t)+p^{\prime}(\omega t) .
\end{aligned}
$$

For a given function $f$, its average value is defined by

$$
F=\bar{f}=(1 / \tau) \int_{t-\tau / 2}^{t+\tau / 2} f(s) d s
$$

so $\mathbf{U}(t)=\overline{\mathbf{u}}, T(t)=\bar{\theta}-\theta_{2}$, and $P(t)=\bar{p}$.

Substituting this decomposition in the momentum equations, we obtain two coupled systems of equations, one for the mean flow and the other for the fluctuating component of the flow. The object of applying the averaging method is then to establish the connection between the oscillatory and mean variables. Let us introduce the additional variable $\mathbf{W}_{\mathbf{T}}$ which constitutes the solenoidal part of the temperature field contribution to the gravitational force in the following Helmholtz decomposition:

$$
\begin{aligned}
& \boldsymbol{\nabla} \wedge T \mathbf{k}=\nabla \wedge \mathbf{W}_{\mathbf{T}}, \\
& \boldsymbol{\nabla} \cdot \mathbf{W}_{\mathbf{T}}=0 .
\end{aligned}
$$

Using the high frequency hypothesis and assuming that the amplitude of displacement is very low, i.e., $b$ $\ll H / \beta_{T}\left(\theta_{1}-\theta_{2}\right)$, the equations of the oscillatory component are linearized according to the procedure developed by $\mathrm{Si}$ monenko et al. ${ }^{26}$ These conditions are valid when $\theta^{\prime}(\omega t)$ $\ll T(t)$. The theoretical justification is given by Simonenko, ${ }^{27}$ where he proves the convergence of solutions of the averaged system to averaged solutions of the system (3)-(5). A numerical description of this convergence is given by Khallouf. ${ }^{28}$ Consequently this allows us to establish the following relationships between mean and fluctuating variables:

$$
\begin{aligned}
& \mathbf{u}^{\prime}=\frac{\operatorname{Ra} R}{A^{2} \omega^{2}+1}(-\sin (\omega t)+A \omega \cos (\omega t)) \mathbf{W}_{\mathbf{T}}, \\
& \theta^{\prime}=-\frac{\operatorname{Ra} R}{A^{2} \omega^{2}+1}\left(\frac{1}{\omega} \cos (\omega t)+A \sin (\omega t)\right) \mathbf{W}_{\mathbf{T}} \cdot \nabla T .
\end{aligned}
$$

The mean motion is then determined by

$$
\begin{aligned}
& \boldsymbol{\nabla} \cdot \mathbf{U}=0, \\
& A \frac{\partial \mathbf{U}}{\partial t}+\mathbf{U}=-\nabla P+\operatorname{Ra} T \mathbf{z}+\operatorname{Ra}_{v} \mathbf{W}_{\mathbf{T}} \cdot \boldsymbol{\nabla}(T \mathbf{k}), \\
& \frac{\partial T}{\partial t}+(\mathbf{U} \cdot \boldsymbol{\nabla}) T=\nabla^{2} T, \\
& \boldsymbol{\nabla} \wedge T \mathbf{k}=\boldsymbol{\nabla} \wedge \mathbf{W}_{\mathbf{T}}, \\
& \boldsymbol{\nabla} \cdot \mathbf{W}_{\mathbf{T}}=0 .
\end{aligned}
$$

We note $\operatorname{Ra}_{v}=\operatorname{Ra}^{2} R^{2} A / 2\left(A^{2} \omega^{2}+1\right)$, the modified vibrational Rayleigh number, is hereafter called the vibrational Rayleigh number. The boundary conditions are in accordance with the physical statement of problems (6)-(9) and

$$
\mathbf{W}_{\mathbf{T}} \cdot \mathbf{n}=0 \quad \text { on } \partial \Omega \text {. }
$$

Note that, for the classical Darcy model, the term $A \partial \mathbf{u} / \partial t$ in Eq. (4) is neglected as $A \approx 10^{-6}$. Eliminating this term leads to wrong mean equations which are independent of the vibration. As one can see, even if $A \approx 10^{-6}$ the vibrational Rayleigh number $\operatorname{Ra}_{v}=\operatorname{Ra}^{2} R^{2} A / 2\left(A^{2} \omega^{2}+1\right)$ can have a finite value.

\section{LINEAR STABILITY}

\section{A. Mechanical equilibrium and stability problem formulation}

An important question is whether or not the state of mechanical equilibrium (i.e., the state at which the mean and 
pulsational velocities are zero) exists in our situation. In order to prove the existence of a conductive solution of the system (17)-(21), we set up $\mathbf{U}=\mathbf{0}, \mathbf{W}_{\mathbf{T}}=\mathbf{0}$ and $\partial / \partial t=0$ in this system and seek a valid solution for arbitrary values of the nondimensional parameters. We obtain the following system:

$$
\begin{aligned}
& \boldsymbol{\nabla} \wedge\left(\operatorname{Ra} T_{0} \mathbf{z}\right)=\mathbf{0}, \\
& \Delta T_{0}=0, \\
& \nabla \wedge T_{0} \mathbf{k}=\mathbf{0},
\end{aligned}
$$

with the following boundary conditions:

$$
\begin{aligned}
& T_{0}=1 \quad \text { for } z=0, \forall x, \\
& T_{0}=0 \quad \text { for } z=1, \forall x, \\
& \frac{\partial T_{0}}{\partial x}=0 \quad \text { for } x=0, \quad A_{L}, \forall z .
\end{aligned}
$$

Equation (24) with boundary conditions (26) and (27) leads to $T_{0}=1-z$.

The conditions (25) lead to $\alpha=\pi / 2$. Consequently in this case we have a strict mechanical equilibrium, i.e., the oscillatory components of the velocity also vanish [see Eqs. (15) and (16)].

In the following, we only deal with the case of vertical vibration.

\section{B. Stability of the equilibrium solution}

It is convenient to rewrite Eqs. (17)-(21) as evolution equations for two-dimensional perturbations superimposed on this equilibrium state. We denote the perturbations by $\left(\mathbf{U}^{\prime}, P^{\prime}, T^{\prime}, \mathbf{W}_{\mathbf{T}}^{\prime}\right)$ and introduce the following streamfunction representations:

$$
\begin{aligned}
& U_{x}^{\prime}=-\partial \psi^{\prime} / \partial z, \quad U_{z}^{\prime}=\partial \psi / \partial x, \\
& W_{T_{x}}^{\prime}=-\partial \psi_{1}^{\prime} / \partial z, \quad W_{T_{z}}^{\prime}=\partial \psi_{1} / \partial x .
\end{aligned}
$$

As a result, a positive streamfunction corresponds to a clockwise cell. Eliminating $P^{\prime}$ we obtain:

$$
\frac{\partial}{\partial t}\left(\begin{array}{c}
-A \Delta \psi^{\prime} \\
T^{\prime} \\
0
\end{array}\right)=\mathbf{L}\left(\begin{array}{c}
\psi^{\prime} \\
T^{\prime} \\
\psi_{1}^{\prime}
\end{array}\right)+\left(\begin{array}{c}
\operatorname{Ra}_{v} N_{1}\left(\psi_{1}^{\prime}, T^{\prime}\right) \\
N_{2}\left(\psi^{\prime}, T^{\prime}\right) \\
0
\end{array}\right),
$$

where for all pairs $(f, g)$ of real functions

$$
\begin{aligned}
& N_{1}(f, g)=\frac{\partial^{2} f}{\partial x \partial z} \frac{\partial g}{\partial x}+\frac{\partial f}{\partial z} \frac{\partial^{2} g}{\partial x^{2}}-\frac{\partial^{2} f}{\partial x^{2}} \frac{\partial g}{\partial z}-\frac{\partial f}{\partial x} \frac{\partial^{2} g}{\partial x \partial z}, \\
& N_{2}(f, g)=\frac{\partial f}{\partial z} \frac{\partial g}{\partial x}-\frac{\partial f}{\partial x} \frac{\partial g}{\partial z}
\end{aligned}
$$

$\mathbf{L}$ is the Jacobian matrix such that

$$
\mathbf{L}=\left(\begin{array}{ccc}
\nabla^{2} & -\operatorname{Ra} \frac{\partial}{\partial x} & \operatorname{Ra}_{v} \frac{\partial^{2}}{\partial x^{2}} \\
\frac{\partial}{\partial x} & \nabla^{2} & 0 \\
0 & -\frac{\partial}{\partial x} & \nabla^{2}
\end{array}\right)
$$

At the boundaries of the cavity the streamfunctions vanish and thus

$$
\begin{aligned}
& \left.\psi^{\prime}\right|_{\partial \Omega}=0, \\
& T^{\prime}(z=0)=T^{\prime}(z=1)=0 \quad \forall x \in\left[0, A_{L}\right], \\
& \frac{\partial T^{\prime}}{\partial x}(x=0)=\frac{\partial T^{\prime}}{\partial x}\left(x=A_{L}\right)=0 \quad \forall z \in[0,1], \\
& \left.\psi_{1}^{\prime}\right|_{\partial \Omega}=0 .
\end{aligned}
$$

Equation (31) with boundary conditions (35)-(38) is invariant under two reflections. These operations are described by the operators $S_{x}, S_{z}$, and $S_{o}=S_{x} S_{z}$ defined by

$$
\begin{aligned}
& S_{x}\left(\begin{array}{l}
\psi^{\prime} \\
T^{\prime} \\
\psi_{1}^{\prime}
\end{array}\right)(x, z)=\left(\begin{array}{c}
-\psi^{\prime} \\
T^{\prime} \\
-\psi_{1}^{\prime}
\end{array}\right)\left(A_{L}-x, z\right), \\
& S_{z}\left(\begin{array}{c}
\psi^{\prime} \\
T^{\prime} \\
\psi_{1}^{\prime}
\end{array}\right)(x, z)=\left(\begin{array}{c}
-\psi^{\prime} \\
-T^{\prime} \\
-\psi_{1}^{\prime}
\end{array}\right)(x, 1-z), \\
& S_{o}\left(\begin{array}{c}
\psi^{\prime} \\
T^{\prime} \\
\psi_{1}^{\prime}
\end{array}\right)(x, z)=\left(\begin{array}{c}
\psi^{\prime} \\
-T^{\prime} \\
\psi_{1}^{\prime}
\end{array}\right)\left(A_{L}-x, 1-z\right),
\end{aligned}
$$

and are generalized reflections since $S_{x}^{2}=1, S_{z}^{2}=1$ and $S_{o}^{2}$ $=1$. Here $S_{x}, S_{z}$, and $S_{x} S_{z}$ represent left-right, up-down, and centrosymmetries, respectively. The representation $\Gamma$ of the group $Z_{2} \times Z_{2}$ is $\Gamma \equiv\left\{\mathrm{Id}, S_{x}, S_{z}, S_{o}\right\}$, where Id is the identity operator and this group plays an important role in the bifurcation analysis described in the following. In particular, it is known that, in the presence of this symmetry group (Crawford and Knobloch, ${ }^{29}$ Golubitsky and Schaeffer, ${ }^{30}$ and Riley and Winters ${ }^{10}$ ), the conduction state can only lose stability to states that are either $S_{x}$ symmetric, $S_{z}$ symmetric, $S_{o}$ symmetric or have all the symmetry properties $\left(S_{o}, S_{x}\right.$, and $S_{z}$ ). The resulting bifurcations are pitchforks except for the last case. Without vibration, it is known that $S_{z}$-symmetric solutions are always unstable and result from at least a second primary bifurcation. The first two primary bifurcations are supercritical pitchforks with either $S_{o}$-symmetric or $S_{x}$-symmetric solutions $\left(\mathrm{Beck}^{4}\right)$. In addition to these symmetries, slip condition (35) implies a translational invariance in the $x$ and $z$ directions. The $(i, j)$ mode corresponding to developments (43)-(45) possesses translational symmetries $T_{i}^{p}$ and $T_{i}^{q}$, where $p=1,2, \ldots, i-1, q=1,2, \ldots, j-1$, and

$$
T_{i}^{p}\left(\begin{array}{c}
\psi^{\prime} \\
T^{\prime} \\
\psi_{1}^{\prime}
\end{array}\right)(x, z)=\left(\begin{array}{c}
\psi^{\prime} \\
T^{\prime} \\
\psi_{1}^{\prime}
\end{array}\right)\left(x+\frac{2 p A_{L}}{i}, z+\frac{2 q}{j}\right) .
$$




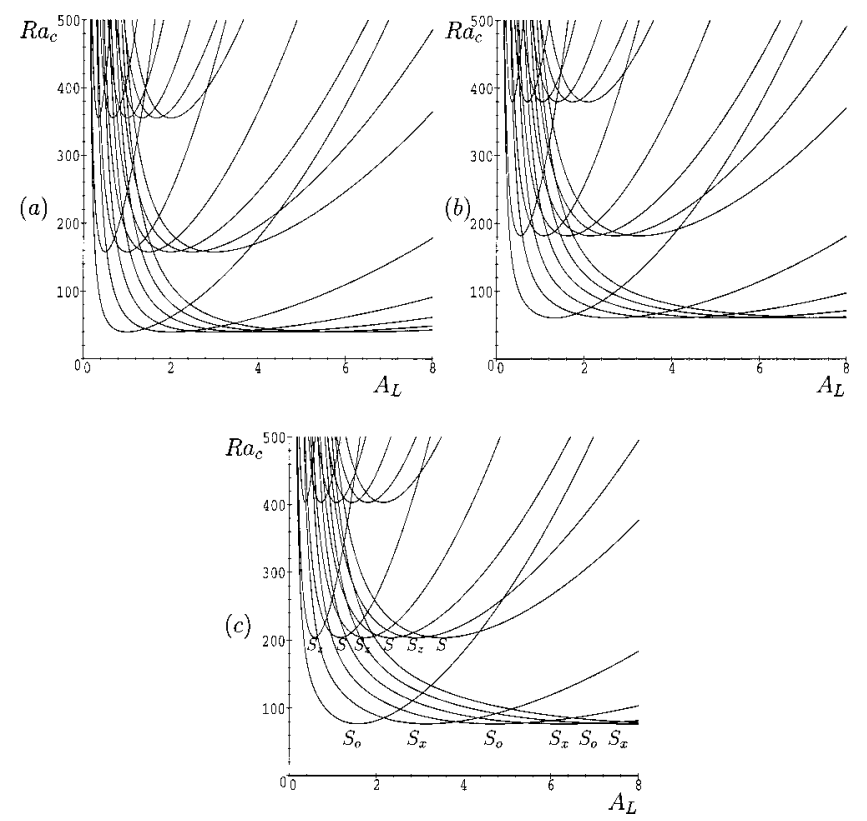

FIG. 2. Critical Rayleigh number $\mathrm{Ra}_{c}$ of a few primary bifurcations vs the aspect ratio $A_{L}$. Only the modes, obtained within the truncation $(n=6, m$ $=3$ ), are represented. (a) Static case $\mathrm{Ra}_{v}=0$. (b) $\mathrm{Ra}_{v}=50$. (c) $\mathrm{Ra}_{v}=100 . \mathrm{S}$ branches possess all the symmetry properties.

This symmetry has an important consequence for the crossing of paths of bifurcation points (see the following). Here, the translational symmetry arising from the slip condition guarantees that the paths corresponding to the $i$ and $i$ +1 modes will cross because these paths break different symmetries ( $S_{o}$ and $S_{x}$ for the first primary bifurcation).

\section{Analytical results}

The linear stability problem is solved by means of a Galerkin method using the following expansions:

$$
\begin{aligned}
& \psi^{\prime}(x, z)=\sum_{i=0}^{n} \sum_{j=0}^{m} a_{i j} \sin \left(i \pi x / A_{L}\right) \sin (j \pi z) e^{I \Omega_{h} t}, \\
& T^{\prime}(x, z)=\sum_{i=0}^{n} \sum_{j=0}^{m} b_{i j} \cos \left(i \pi x / A_{L}\right) \sin (j \pi z) e^{I \Omega_{h} t}, \\
& \psi_{1}^{\prime}(x, z)=\sum_{i=0}^{n} \sum_{j=0}^{m} c_{i j} \sin \left(i \pi x / A_{L}\right) \sin (j \pi z) e^{I \Omega_{h} t},
\end{aligned}
$$

where $\Omega_{h}$ is a real number, $I=\sqrt{-1}$, and $(i, j) \in \mathbb{N}^{2}$.

The $S_{x}$-symmetric eigenmodes contain an even number of rolls in the $x$ direction whereas the $S_{z}$-symmetric ones contain an even number of rolls in the $z$ direction. The number of cells is linked with the values $i$ and $j$, i.e., $i$ is the number of cells along the $x$ axis and $j$ along the $z$ axis. The $S_{o}$-symmetric eigenmodes contain an odd number of cells in both directions. The eigenmodes which possess all the symmetry properties contain an even number of cells in both directions. For $j=1$ (first primary bifurcation), the eigenvector could be either $S_{x}$ symmetric or $S_{o}$ symmetric. So, for $j$ $=2$ (second primary bifurcation), the eigenvector could be either $S_{z}$ symmetric or have all the symmetry properties $\left(S_{o}\right.$, $S_{x}$, and $S_{z}$ ).
The bifurcation points are located by solving the linear algebraic system $£ \mathbf{X}=0$ with $\mathbf{X}=\left(a_{i j}, b_{i j}, c_{i j}\right)_{i=0, n ; j=0, m}$ and $£$ is evaluated by the Galerkin method.

For particular values $\left(\mathrm{Ra}, \Omega_{h}\right.$ ), the determinant of $£$ is zero, indicating a bifurcation point. We find that

$$
\begin{aligned}
\operatorname{det}(£)= & \prod_{i=1}^{n} \prod_{j=1}^{m}\left[f\left(A, \mathrm{Ra}, \mathrm{Ra}_{v}, \Omega_{h}, A_{L}, i, j\right)+I \Omega_{h}\left(A_{L}^{2} j^{2}\right.\right. \\
& \left.\left.+i^{2}\right)^{2}\left(\pi^{2} A_{L}^{2} j^{2} A+A_{L}^{2}+\pi^{2} i^{2} A\right)\right]
\end{aligned}
$$

with $f$ a real function. As $\operatorname{det}(\mathfrak{E})=0$ only if both its real part and its imaginary part vanish, this leads to $\Omega_{h}=0$. The principle of stability exchange applies for this problem even if the linear problem it not self-adjoint.

The critical thermal Rayleigh number which determines the limit of the even and odd solutions can be studied analytically by

$$
\mathrm{Ra}_{c}=\frac{\pi^{2}\left(i^{2}+j^{2} A_{L}^{2}\right)^{3}+i^{4} A_{L}^{2} \mathrm{Ra}_{v}}{i^{2} A_{L}^{2}\left(i^{2}+j^{2} A_{L}^{2}\right)}
$$

The graphs of the primary bifurcation points are shown in Fig. 2. The cellular modes $\left(i_{\text {odd }}, j_{\text {odd }}\right)$ are $S_{o}$ symmetric, $\left(i_{\text {odd }}, j_{\text {even }}\right)$ are $S_{x}$ symmetric, $\left(i_{\text {even }}, j_{\text {odd }}\right)$ are $S_{z}$ symmetric, and $\left(i_{\text {even }}, j_{\text {even }}\right)$ possess all of these aforementioned symmetries (see Fig. 3). If $\mathrm{Ra}_{v}$ increases, the curves of the critical Rayleigh number are both translated up and right and rescaled (Fig. 2) but keep the same shape.

\section{NONLINEAR ANALYSIS}

Weakly nonlinear analysis. In this section we use a multiple scale analysis to reduce system (31) to a simpler form, called a normal form, valid near onset of the first primary instability, i.e., for $\left|R a-R a_{c}\right| / R_{c} \ll 1$. We expand the perturbations, the Rayleigh number, and time in powers of a small parameter $\epsilon>0$ :

$$
\begin{aligned}
& \psi^{\prime}=\epsilon \psi^{(1)}+\epsilon^{2} \psi^{(2)}+\cdots, \\
& T^{\prime}=\epsilon T^{(1)}+\epsilon^{2} T^{(2)}+\cdots, \\
& \psi_{1}^{\prime}=\epsilon \psi_{1}^{(1)}+\epsilon^{2} \psi_{1}^{(2)}+\cdots, \\
& \frac{\partial}{\partial t}=\epsilon \frac{\partial}{\partial t^{(1)}}+\epsilon^{2} \frac{\partial}{\partial t^{(2)}}+\cdots, \\
& \operatorname{Ra}=\operatorname{Ra}_{c}+\epsilon \mathrm{Ra}^{(1)}+\epsilon^{2} \mathrm{Ra}^{(2)}+\cdots
\end{aligned}
$$

At order $\epsilon$ we recover the linear eigenvalue problem. The perturbation eigenmodes can be written in the form 


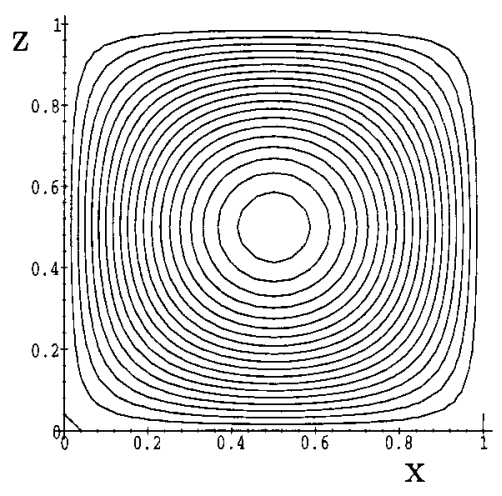

(a)

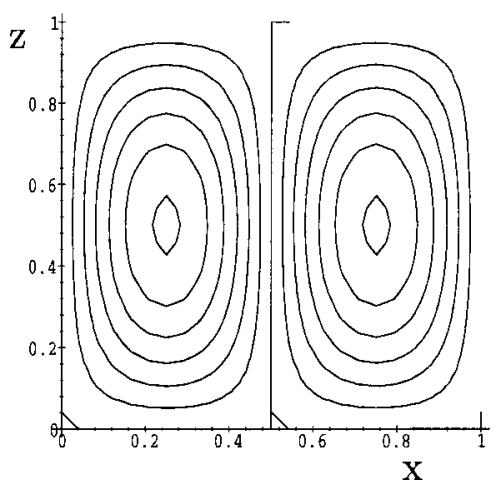

(c)

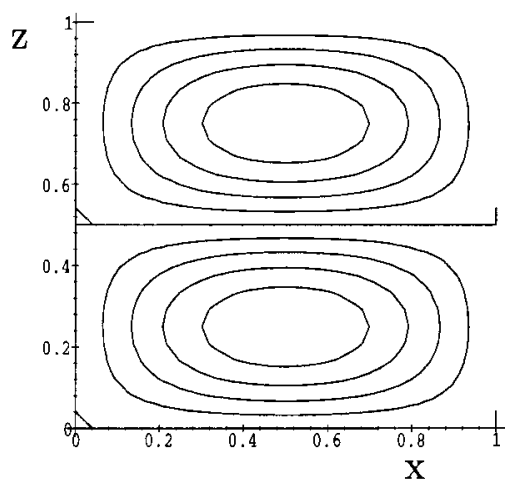

(b)

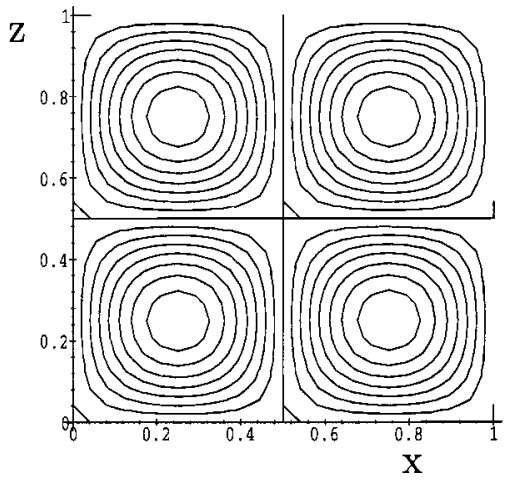

(d)
FIG. 3. Streamfunction for $A_{L}=1$ in the static case. (a) $S_{o}$-symmetric solution. (b) $S_{z}$-symmetric solution, (c) $S_{x}$-symmetric solution, and (d) solution with all the symmetry properties.

$$
\begin{aligned}
\left(\begin{array}{l}
\psi^{(1)} \\
T^{(1)} \\
\psi_{1}^{(1)}
\end{array}\right)= & K\left(t^{(k)}\right) \\
& \times\left(\begin{array}{c}
\frac{\pi^{2}\left(j^{2} A_{L}^{2}+i^{2}\right)^{2}}{i^{2} A_{L}^{2}} \sin \left(i \frac{\pi x}{A_{L}}\right) \sin (j \pi z) \\
\frac{\pi^{2}\left(j^{2} A_{L}^{2}+i^{2}\right)}{i A_{L}} \cos \left(i \frac{\pi x}{A_{L}}\right) \sin (j \pi z) \\
\sin \left(i \frac{\pi x}{A_{L}}\right) \sin (j \pi z)
\end{array}\right),
\end{aligned}
$$

where the amplitude $K$ depends on the slow times $t^{(k)}, k$ $=1,2, \ldots$. We note that for moderate values of $\mathrm{Ra}_{v}$, whatever the aspect ratio value, the mode of lowest critical Rayleigh number always has unit vertical wave number, while the corresponding horizontal wave number is dependent both on the aspect ratio and on $\mathrm{Ra}_{v}$. We will see later the meaning of moderate. The wave number of this mode changes from $i$ to $i+1$ at $A_{L}=A_{L_{i}}$ (Let us say that $\left.A_{L_{0}}=0\right)$. In the static case ${ }^{10}$ $A_{L_{i}}=(i(i+1))^{1 / 2}$ but this relation does not hold with vibration. The critical Rayleigh number associated with the first primary bifurcation point for moderate values of $\mathrm{Ra}_{v}$ is

$$
\mathrm{Ra}_{c}=\frac{\pi^{2}\left(i^{2}+A_{L}^{2}\right)^{3}+i^{4} A_{L}^{2} \mathrm{Ra}_{v}}{i^{2} A_{L}^{2}\left(i^{2}+A_{L}^{2}\right)}
$$

for $A_{L_{i-1}}<A_{L}<A_{L_{i}}$. Figure 4 shows the variation of these analytical curves for increasing $\mathrm{Ra}_{v}$. The vibrations stabilize the diffusive solution and the onset of motion occurs for larger values of the Rayleigh number. $\forall i,\left|A_{L_{i}}-A_{L_{i-1}}\right|$ $=f\left(\mathrm{Ra}_{v}, i\right)$ is the length of box for which the number of cells

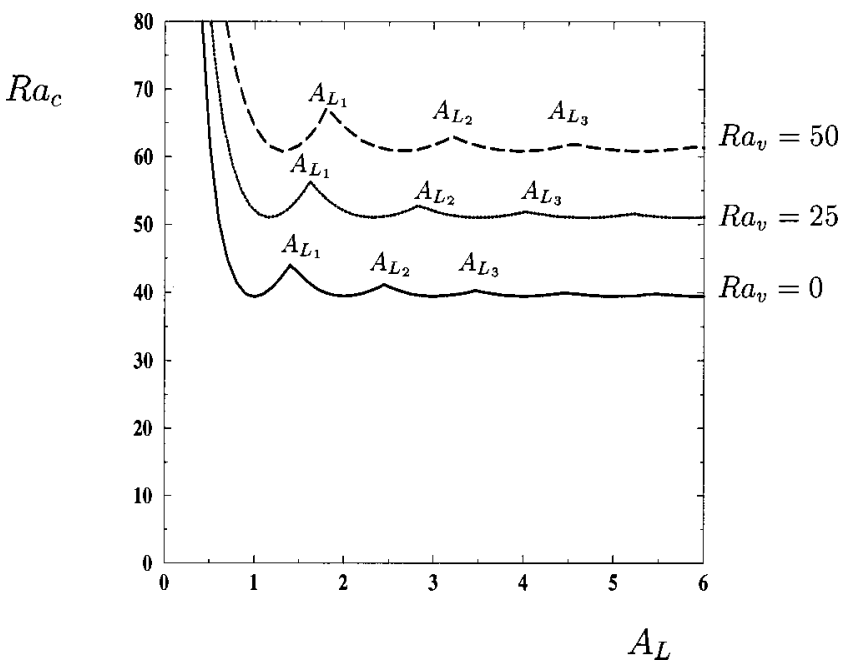

FIG. 4. Critical Rayleigh number $\mathrm{Ra}_{c}$ of the first primary bifurcation point vs the aspect ratio $A_{L}$. The vertical vibrations increase the value of the critical Rayleigh number. For $A_{L}<A_{L_{1}} S_{o}$-symmetric one-cell flow, for $A_{L_{1}}<A_{L}<A_{L_{2}} S_{x}$-symmetric two-cell flow. For $A_{L_{i}}<A_{L}<A_{L_{i+1}}(i+1)$-cell flow which is $S_{o}$ symmetric for $i$ odd or $S_{x}$ symmetric for $i$ even. 
increases by one. Consequently, $\pi / f\left(\mathrm{Ra}_{v}, i\right)$ approximates the wave number of the infinite horizontal layer. In the static case $f(0, i) \approx 1$, which corresponds to the well-known wave number of $\pi$. The important thing is that vertical vibration can generate a one-cell flow whatever the value of $A_{L}$ as $f\left(\mathrm{Ra}_{v}\right) \rightarrow \infty$ when $\mathrm{Ra}_{v} \rightarrow \infty$. For a finite value of $A_{L}$ there is a finite value $\mathrm{Ra}_{v c}$ of $\mathrm{Ra}_{v}$ such that the preferred mode has a one-cell structure. This upper limit $\operatorname{Ra}_{v c}=\pi^{2}\left(1+A_{L}^{2}\right)\left(20 A_{L}^{4}\right.$ $\left.+13 A_{L}^{2}+2\right) / A_{L}^{2}$ defines the upper limit of the moderate domain of $\mathrm{Ra}_{v}$.

For $\mathrm{Ra}_{v}>\mathrm{Ra}_{v c}$, the preferred mode is the $(1, j)$ mode with $j \geqslant 2$ and the critical Rayleigh number value associated with the preferred mode is given by Eq. (47) with $i=1$ and $j \geqslant 2$. The mode of lowest critical Rayleigh number always has unit horizontal wave number, while the corresponding vertical wave number is then dependent on $\mathrm{Ra}_{v}$. The surprising thing is that increasing $\mathrm{Ra}_{v}$ will also increase the value $j$ of the preferred mode which means that stacked rolls $\left(S_{z}\right.$ or $S_{o}$-symmetric solutions) are then stable solutions at the onset of convection. This type of symmetry has not been observed in convection experiments but this system allows experimental investigation of this state. On earth, we need to impose a vibrational field intensity which is bigger than $10 \mathrm{~g}$. We should mention that this type of flow could be easily observed by a space experiment for which the gravity will be strongly reduced.

In the following, only moderate, and also more realistic, values of $\mathrm{Ra}_{v}$ are considered.

At order $\epsilon^{2}$, whatever the value of the aspect ratio $A_{L}$, the quadratic terms in the amplitude equation necessarily vanish and we can set $\mathrm{Ra}^{(1)}=0$ and omit the time scale $t^{(1)}$.

At order $\epsilon^{3}$, the existence of a convective solution requires the solvability lemma ${ }^{31}$ to be satisfied, i.e., there must be a nonzero solution to the following adjoint linear eigenvalue problem:

$$
\left(\begin{array}{ccc}
\nabla^{2} & -\frac{\partial}{\partial x} & 0 \\
\operatorname{Ra} \frac{\partial}{\partial x} & \nabla^{2} & \frac{\partial}{\partial x} \\
\operatorname{Ra}_{v} \frac{\partial}{\partial x^{2}} & 0 & \nabla^{2}
\end{array}\right)\left(\begin{array}{l}
\psi^{*} \\
T^{*} \\
\psi_{1}^{*}
\end{array}\right)=\left(\begin{array}{l}
0 \\
0 \\
0
\end{array}\right),
$$

with identical boundary conditions. This condition yields the result

$$
\mathrm{Ra}_{c}^{*}=\mathrm{Ra}_{c} .
$$

The amplitude equation (57) now follows on applying the Fredholm alternative

$$
a \frac{\partial K}{\partial t^{(2)}}=b K\left(\mu+c K^{2}\right),
$$

with $\mu=\left(\mathrm{Ra}-\mathrm{Ra}_{c}\right) / \epsilon^{2}$ the bifurcation parameter. This amplitude equation applies for $A_{L_{i-1}}<A_{L}<A_{L_{i}}$ and the coefficients $a, b$, and $c$ are evaluated analytically:

$$
a=\frac{\pi^{4} A\left(i^{2}+A_{L}^{2}\right)^{3}+\pi^{2}\left(A_{L}+i I\right)^{2}\left(A_{L}-i I\right)^{2} A_{L}^{2}}{4 i^{2} A_{L}^{3}},
$$

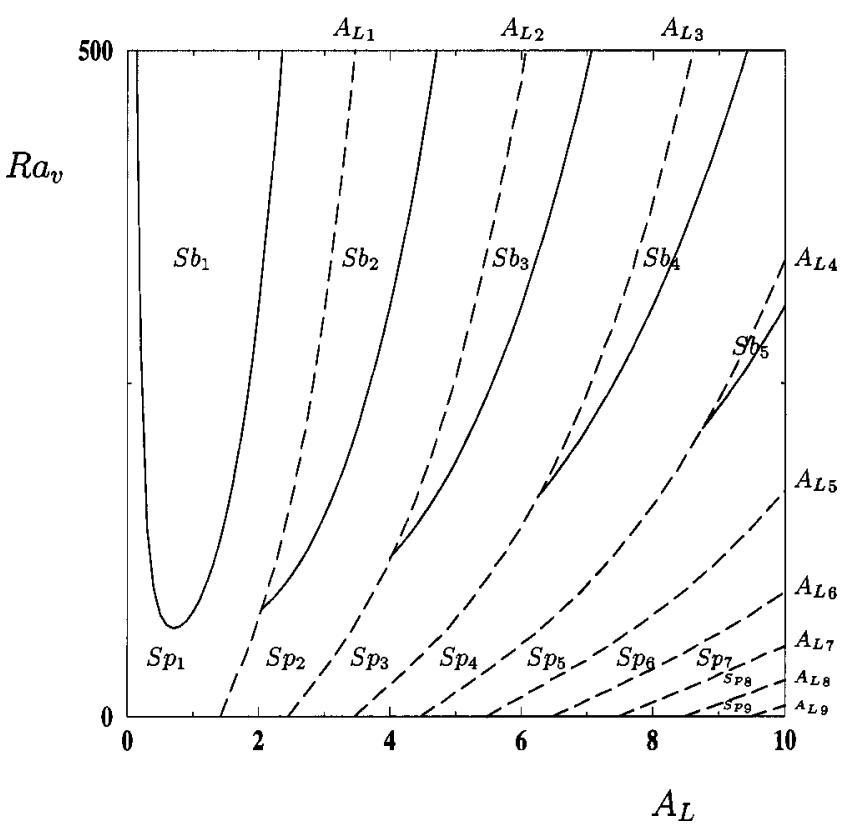

FIG. 5. For a chosen pair $\left(A_{L}-\mathrm{Ra}_{v}\right)$ the first primary bifurcation could be a supercritical stable pitchfork $(\mathrm{Sp})$ or subcritical unstable pitchfork $(\mathrm{Sb})$. The subscript $\left(\mathrm{Sp}_{i}\right.$ or $\left.\mathrm{Sb}_{i}\right)$ indicates the number, $i$, of cells on the emerging branch of the solution. As previously mentioned, $i$ odd means the solution is $S_{o}$ symmetric and $i$ even the solution is $S_{x}$ symmetric $(j=1)$.

$$
\begin{aligned}
& b=\frac{i^{2}+A_{L}^{2}}{4 A_{L}}, \\
& c=\frac{\operatorname{Ra}_{v} \pi^{6}\left(i^{2}+A_{L}^{2}\right)^{2}}{8 A_{L}^{4}}-\frac{\pi^{8}\left(i^{2}+A_{L}^{2}\right)^{5}}{8 i^{4} A_{L}^{6}} .
\end{aligned}
$$

For $A_{L}=A_{L_{i}}$ a codimension two bifurcation results from the interaction between $S_{o}$ - and $S_{x}$-symmetric solutions. The mode interaction is not the purpose of the present paper but the analysis is currently under way.

When $c<0$ (respectively, $c>0$ ), the bifurcation is supercritical and stable (respectively, subcritical and unstable) as $-b \mu / a<0$ (respectively, $-b \mu / a>0$ ). Whatever the mode considered and the aspect ratio, $c$, which has a negative value for $\mathrm{Ra}_{v}=0$, becomes positive as $\mathrm{Ra}_{v}$ increases. This occurs within the moderate range of $\mathrm{Ra}_{v}$. Figure 5 represents the different scenarios occurring at the onset of convection and depending on both the aspect ratio and $\mathrm{Ra}_{v}$.

\section{NUMERICAL RESULTS}

\section{A. Numerical method}

The system of equations (17)-(21) was solved numerically using a spectral method. ${ }^{32}$ The method used is based on the projection diffusion algorithm developed by Khallouf. ${ }^{24}$ Temporal integration consists of a semi-implicit secondorder finite difference approximation and transforms the system into a Helmholtz problem arising from the advectiondiffusion equations (19) coupled to the Poisson problems (20) and (21) with appropriate boundary conditions. The latter are solved using the Uzawa (or Poisson-type) formulation of Azaiez et al. ${ }^{33}$ All the subproblems obtained are either Helmholtz or Poisson-type operators. A high-accuracy spec- 


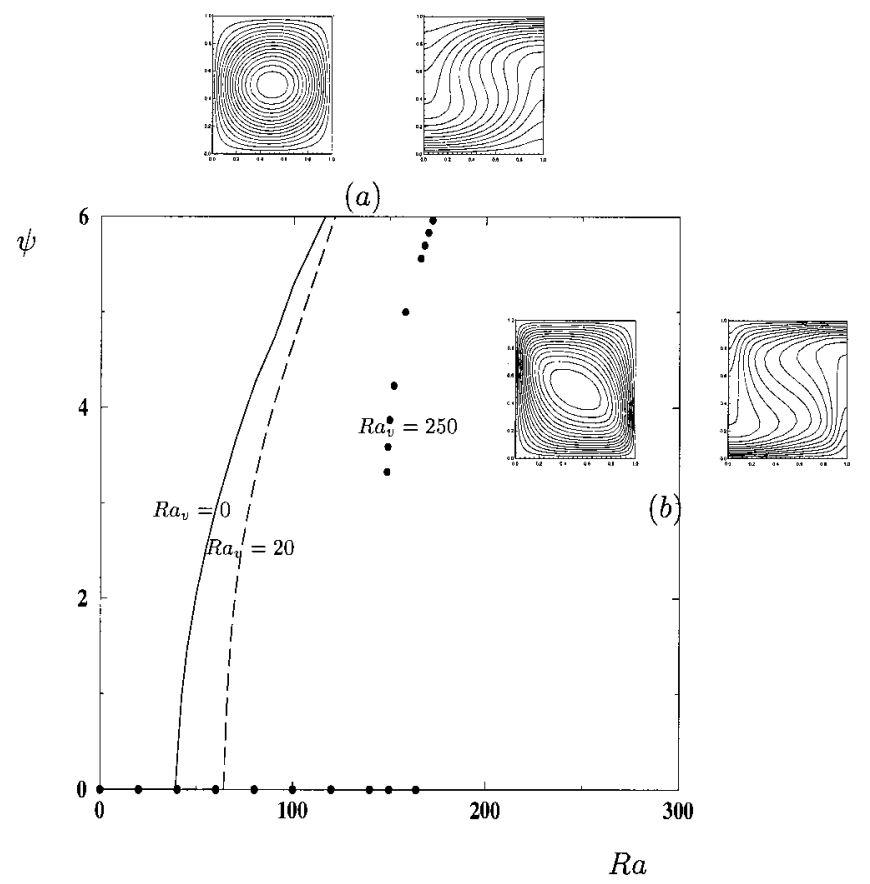

FIG. 6. Bifurcation diagram in the $\psi(0.5,0.5)-\mathrm{Ra}$ plane for different values of $\operatorname{Ra}_{v}$ when $A_{L}=1$. The insets are streamlines and lines of constant mean temperature at $\mathrm{Ra}=165$ for $\mathrm{Ra}_{v}=0$ (a) or $\mathrm{Ra}_{v}=250$ (b). Only the branch corresponding to the preferred mode is represented. Resolution is $33 \times 33$.

tral method, namely one utilizing Legendre collocation points, solves the Helmholtz or Poisson-type operators. Successive diagonalization is used to invert these operators. We mention that the Darcy-Euler solvers are direct and guarantee an accurate spectral solution with divergence-free solenoidal fields over the entire domain, including the boundaries. For the calculations discussed in this paper, a grid with $25 \times 25$ mesh points in the $(x, z)$ domain accurately describes the flow. We selected a $33 \times 33$ grid for our calculations.

\section{B. Numerical results}

For the numerical study, we chose $A_{L}=1$ or $A_{L}=3$ in order to investigate cases in which there are substantially different effects of vibration (see Fig. 5). The numerical simulations were done as follows: The vibrational Rayleigh number was fixed and we increased the value of the thermal Rayleigh number. The following results apply in our computational domain, i.e., $0<\mathrm{Ra}<300$ and $0<\mathrm{Ra}_{v}<400$.

\section{Square cavity $A_{L}=1$}

The bifurcation diagram is presented in Fig. 6. In the static case, the pitchfork bifurcation point occurs at $\mathrm{Ra}$ $\approx 4 \pi^{2}$ and the emerging branch is supercritical and stable. Along this branch, the solution is a one-cell flow [see Fig. 6(a)], i.e., $S_{o}$ symmetric for the range of Rayleigh numbers investigated. By increasing the vibrational Rayleigh number, we delay the onset of convection but the pitchfork remains supercritical up to $\mathrm{Ra}_{v}=80$. For $\mathrm{Ra}_{v}>80$, the vibration destabilizes this pitchfork branch which becomes subcritical, i.e., unstable. The subcritical branch undergoes a saddlenode bifurcation and becomes stable. Along this stable branch, the solutions consist of symmetric one-roll flow

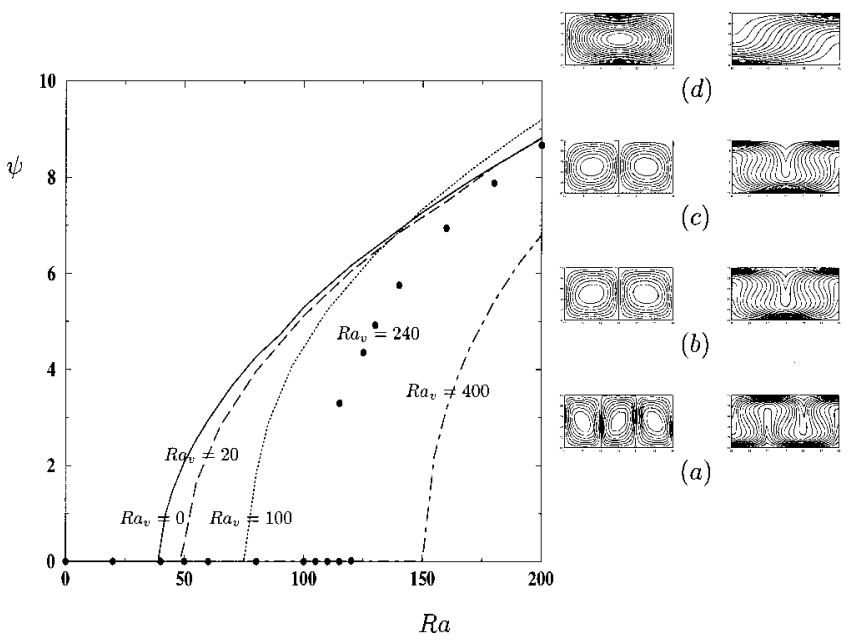

FIG. 7. Bifurcation diagram in the $\psi(0.5,0.5)-\mathrm{Ra}$ plane for different values of $\mathrm{Ra}_{v}$ when $A_{L}=3$. The insets are streamlines and lines of constant mean temperature at $\mathrm{Ra}=165$ for $\mathrm{Ra}_{v}=20$ (a), $\mathrm{Ra}_{v}=100$ (b), $\mathrm{Ra}_{v}=240$ (c), and $\mathrm{Ra}_{v}=400$ (d). Only the branch corresponding to the preferred mode is represented. Resolution is $33 \times 33$.

structures [see Fig. 6(b)]. Whatever the value of $\mathrm{Ra}_{v}>80$, the first primary bifurcation remains a subcritical pitchfork.

\section{A rectangular cavity $A_{L}=3$}

Figure 7 presents the bifurcation diagram for different values of $\mathrm{Ra}_{v}$. In this case, the situation differs from the previous one. As presented in Fig. 5, the type of flow at the onset of convection is centrosymmetric but possesses a three-cell structure in the static case [see Fig. 7(a)]. When $\mathrm{Ra}_{v}$ increases up to $\mathrm{Ra}_{v}=40$, the conduction solution remains stable up to a larger value of the Rayleigh number. The emerging branch is a supercritical pitchfork along which the solutions have a three-cell structure i.e., $S_{o}$ symmetric. For $40<\mathrm{Ra}_{v}<160$, the emerging branch remains a supercritical pitchfork but the solutions have a two-cell structure, i.e., $S_{x}$ symmetric [see Fig. 7(b)]. For $160<\mathrm{Ra}_{v}<350$, the pitchfork bifurcation becomes subcritical and the emerging branch is unstable. This branch undergoes a saddle-node bifurcation and becomes stable. Along this stable branch, the solutions consist of two-cell flow structures [see Fig. 7(c)]. For $\operatorname{Ra}_{v}>350$ up to the end of our computational domain, the first primary bifurcation becomes supercritical but the solutions along this branch then have a one-cell structure [see Fig. 7(d)]. For $\operatorname{Ra}_{v}>1250$ the pitchfork bifurcation becomes subcritical.

Increasing the strength of vibration will increase the value of the critical Rayleigh number and in this sense stabilize the diffusive solution. In fact the vibration imposes lines of constant temperature perpendicular to the axis of vibration, which in our present will be study horizontal and the horizontal profile of these lines defines the restless state. The variations of these lines are shown by in Figs. 6(a) and 6(b) or Figs. 7(a)-7(d).

Moreover, a sufficiently high strength of vibration forces the motion to have a one-cell flow structure at the onset of convection. 


\section{CONCLUSION}

Analytical and numerical techniques were used to study the linear stability of Lapwood convection in a twodimensional cavity subjected to vibration. The term $A \partial \mathbf{u} / \partial t$ in the Darcy model cannot be neglected when the cavity medium is subjected to linear harmonic high-frequency oscillations. A pure mechanical equilibrium exists only for vertical vibrations which stabilize the no-flow state. More precisely, vibrations move the onset of convection to larger values of the Rayleigh number. The surprise is that vertical vibrations can generate a one-cell mode at the onset of convection whatever the value of the aspect ratio. This is done by crossing an appropriate number of codimension two bifurcation points.

A natural extension of the present study will analyze the effect of vertical vibration on the primary and secondary bifurcation points, i.e., on the modal exchange mechanisms in Lapwood convection.

\section{ACKNOWLEDGMENTS}

This work would not have been possible without the support of C.N.E.S. (Centre National d'Etudes Spatiales). We thank G. Pinaud for his work and his contribution to the present study. We also thank P. Brancher for his careful reading and $\mathrm{S}$. Becker for checking the English.

${ }^{1}$ S. A. Bories, "Natural convection in porous media," Proceedings NATO ASI on Fundamental of Transport Phenomena in Porous Media, 1985 (unpublished).

${ }^{2}$ D. A. Nield and A. Bejan, Convection in Porous Media (Springer, Berlin, 1992).

${ }^{3}$ G. Z. Gershuni and D. V. Lyubimov, Thermal Vibrational Convection (Wiley, New York, 1998).

${ }^{4}$ J. L. Beck, "Convection in a box of porous material saturated with fluid," Phys. Fluids 15, 1377 (1972).

${ }^{5}$ J. M Straus and G. Schubert, "On the existence of three-dimensional convection in a rectangular box containing fluid-saturated porous material,'” J. Fluid Mech. 87, 385 (1978).

${ }^{6}$ J. M. Straus and G. Schubert, "Three-dimensional convection in a cubic box of fluid-saturated material,' J. Fluid Mech. 91, 155 (1979).

${ }^{7}$ J. M. Straus and G. Schubert, "Modes of finite-amplitude threedimensional convection in rectangular boxes of fluid-saturated porous material," J. Fluid Mech. 103, 23 (1981).

${ }^{8}$ G. Schubert and J. M. Straus, "Three-dimensional and multi-cellular steady and unsteady convection in fluid-saturated porous media at high Rayleigh numbers," J. Fluid Mech. 94, 25 (1979).

${ }^{9}$ J. P. Caltagirone, G. Meyer, and A. Mojtabi, "Structurations thermoconvectives tridimensionnelles dans une couche poreuse horizontale," J. Mec. 20, 219 (1981).
${ }^{10}$ D. S. Riley and K. H. Winters, "Modal exchange mechanisms in Lapwood convection,"' J. Fluid Mech. 204, 325 (1989).

${ }^{11}$ J. I. D. Alexander, "Residual gravity jitter effects on fluid processes," Microgravity Sci. Technol. 7, 131 (1994).

${ }^{12}$ G. Z. Gershuni and E. M. Zhukhovitsky, "Vibrational thermal convection in zero-gravity," Fluid Mech.-Sov. Res. 15, 63 (1986).

${ }^{13}$ P. M. Gresho and R. L. Sani, "The effects of gravity modulation on the stability of a heated fluid layer," J. Fluid Mech. 40, 783 (1970).

${ }^{14}$ S. Rosenblat and G. A. Tanaka, "Modulation of thermal convection instability," Phys. Fluids 14, 1319 (1971).

${ }^{15}$ M. N. Roppo, S. H. Davis, and S. Rosenblat, "Benard convection with time-periodic heating," Phys. Fluids 27, 796 (1984).

${ }^{16}$ G. Halers, P. Hohenberg, and P. C. Lucke, "Thermal convection under external modulation of the driving force. I. The Lorenz model," Phys. Rev. A 32, 3493 (1985).

${ }^{17} \mathrm{P}$. Hohenberg and J. B. Swift, "Hexagons and rolls in periodically modulated Rayleigh-Benard convection," Phys. Rev. A 35, 3855 (1987).

${ }^{18}$ G. Halers, P. Hohenberg, and P. C. Lucke, "Thermal convection under external modulation of the driven force. II. Experiments," Phys. Rev. A 32, 3519 (1985)

${ }^{19}$ J. J. Niemela and R. J. Donnelly, "External modulation of RayleighBenard convection,’’ Phys. Rev. Lett. 59, 2431 (1987).

${ }^{20}$ G. Bardan, E. Knobloch, A. Mojtabi, and H. Khallouf, "Natural doubly diffusive convection with vibration," Fluid Dyn. Res., preprint.

${ }^{21}$ A. Farooq and G. M. Homsy, "Streaming flows due to $g$-jitter-induced natural convection," J. Fluid Mech. 271, 351 (1994).

${ }^{22}$ P. Grassia and G. M. Homsy, "Buoyant flows with low-frequency jitter," Phys. Fluids 10, 1903 (1998).

${ }^{23}$ Z. Wenfei and L. Xiaojiang, "Optimum source frequency in vibration production technology,"' J. Pet. Sci. Eng. 21, 239 (1998).

${ }^{24}$ H. Khallouf, G. Z. Gershuni, and A. Mojtabi, "Some properties of convective oscillations in porous medium," Numer. Heat Transfer, Part A 30, 605 (1996).

${ }^{25}$ N. N. Bogljubov and Yu. A. Mittropolskil, "Asymptotic methods in the theory of nonlinear oscillations," English translation, International Monographs on Advanced Mathematics and Physics, Hindustan, Dehli, 1962 (unpublished).

${ }^{26}$ I. B. Simonenko and S. M. Zen'kovskaja, "On the effect of high frequency vibrations on the origin of convection," Izv. Akad. Nauk SSSR, Mekh. Zhidk. Gaza 5, 51 (1966).

${ }^{27}$ I. B. Simonenko, "A justification of the averaging method for a problem of convection in a field rapidly oscillating forces and other parabolic equations," Mat. Sb. 2, 245 (1972).

${ }^{28} \mathrm{H}$. Khallouf, "Simulation numérique de la convection thermovibrationnelle par une méthode spectrale," These de doctorat de l'universite Paul Sabatier Toulouse III, 1995.

${ }^{29}$ J. D. Crawford and E. Knobloch, "Symmetry and symmetry-breaking bifurcation in fluid dynamics," Annu. Rev. Fluid Mech. 23, 341 (1991).

${ }^{30}$ M. Golubitsky and D. G. Schaeffer, Singularities and Groups in Bifurcation Theory (Springer, New York, 1985), Vol. 1.

${ }^{31}$ D. D. Joseph and D. H. Sattinger, "Bifurcating time periodic solutions and their stability," Arch. Ration. Mech. Anal. 69, 79 (1972).

${ }^{32}$ C. Canuto, M. Y. Hussaini, A. Quarteroni, and T. A. Zang, Spectral Methods in Fluid Dynamics (Springer, New York, 1987).

${ }^{33}$ M. Azaiez, C. Bernardi, and M. Grundmann, "Spectral methods applied to porous media," East-West J. Numer. Math. 2, 91 (1994). 